Теорія Ймовір. та Матем. Статист. Вип. 75, 2006
Theor. Probability and Math. Statist. No. 75, 2007, Pages 147-160 S 0094-9000(08)00721-7

Article electronically published on January 25, 2008

\title{
GENERALIZED DIFFERENTIABILITY WITH RESPECT TO THE INITIAL DATA OF A FLOW GENERATED BY A STOCHASTIC EQUATION WITH REFLECTION
}

UDC 519.21

\author{
A. YU. PILIPENKO
}

Abstract. Let $\varphi_{t}(x), x \in \mathbb{R}_{+}^{d}$, be a solution of a stochastic differential equation in the half-space $\mathbb{R}_{+}^{d}$ with normal reflection in the boundary; the solution starts from a point $x$. We prove that the random mapping $\varphi_{t}(\cdot, \omega)$ is differentiable in the Sobolev sense for almost all $\omega$. We obtain a stochastic equation for the derivative $\nabla \varphi_{t}$.

\section{INTRODUCTION}

Let $\varphi_{t}(x), x \in \mathbb{R}_{+}^{d}=\mathbb{R}^{d-1} \times[0, \infty)$, be a solution of the following stochastic differential equation in $\mathbb{R}_{+}^{d}$ with normal reflection in the boundary:

$$
\left\{\begin{array}{l}
d \varphi_{t}(x)=a_{0}\left(\varphi_{t}(x)\right) d t+\sum_{k=1}^{m} a_{k}\left(\varphi_{t}(x)\right) d w_{k}(t)+\bar{n} \xi(x, d t), \quad t \geq 0, \\
\varphi_{0}(x)=x, \xi(x, 0)=0, x \in \mathbb{R}_{+}^{d},
\end{array}\right.
$$

where $\bar{n}=(0, \ldots, 0,1) ; \xi(x, t)$ is not decreasing with respect to $t$ for a fixed $x$ and is increasing at the points for which $\varphi_{t}(x) \in \mathbb{R}^{d-1} \times\{0\}$ :

$$
\xi(x, t)=\int_{0}^{t} \mathbb{1}_{\left\{\varphi_{s}(x) \in \mathbb{R}^{d-1} \times\{0\}\right\}} \xi(x, d s) .
$$

It is well known [3] that if the coefficients of equation (1) are Lipschitz functions, then a unique strong solution of (1) exists for all fixed $x \in \mathbb{R}_{+}^{d}$. Applying the Kolmogorov theorem on the existence of a continuous version, one can check (similarly to the case of stochastic differential equations without reflection) that a version of the processes $\varphi$ and $\xi$ that is continuous with respect to $(t, x)$ exists (see, for example, [1]). It is proved in the paper [2] that, for almost all $\omega$ and all $t \geq 0$, the random mapping $\varphi_{t}: \mathbb{R}_{+}^{d} \rightarrow \mathbb{R}_{+}^{d}$ belongs to the intersection of the Sobolev spaces

$$
\bigcap_{p>1} W_{p, \text { loc }}^{1}\left(\mathbb{R}_{+}^{d}, \mathbb{R}^{d}\right)
$$

The aim of this paper is to establish stochastic equations for the derivative $\nabla \varphi_{t}$.

Note that $\varphi_{t}$ is not necessarily differentiable with respect to $x$ (in the usual sense) if the coefficients of equation (1) are smooth. Indeed, consider an example where $d=1$,

2000 Mathematics Subject Classification. Primary 60H10; Secondary 60J25, 60F25.

Key words and phrases. Stochastic equations with reflection, stochastic flows, Sobolev spaces.

Supported by the Ministry for Science and Education of Ukraine, project GP/F8/0086. 
$a_{1} \equiv 1$, and all other coefficients are zero, that is, $\varphi_{t}(x)$ is a Brownian motion with reflection. It is easy to check in this case that

$$
\varphi_{t}(x)= \begin{cases}x+w(t), & x+\min _{0 \leq s \leq t} w(s)>0, \\ w(t)-\min _{0 \leq s \leq t} w(s), & x+\min _{0 \leq s \leq t} w(s) \leq 0 .\end{cases}
$$

The function $\varphi_{t}$ is not differentiable at the point $x=-\min _{0 \leq s \leq t} w(s)>0$. However, it is continuous and belongs to $W_{p, \text { loc }}^{1}\left(\mathbb{R}_{+}\right)$. Moreover

$$
\nabla \varphi_{t}(x)= \begin{cases}1, & x+\min _{0 \leq s \leq t} w(s)>0 \\ 0, & x+\min _{0 \leq s \leq t} w(s)<0 .\end{cases}
$$

The equation for the Sobolev derivative $\nabla \varphi_{t}$ is not a "classical" stochastic differential equation. The equation for $\nabla \varphi_{t}$ can be described informally in the following way.

Let $\varphi_{t}(x) \notin \mathbb{R}^{d-1} \times\{0\}$ and

$$
\tau_{t}(x)=\inf \left\{s \geq t: \varphi_{s}(x) \in \mathbb{R}^{d-1} \times\{0\}\right\} .
$$

Then the derivative $\nabla \varphi_{s}(x)$ satisfies the linear equation

$$
d \nabla \varphi_{s}(x)=\nabla a_{0}\left(\varphi_{s}(x)\right) \nabla \varphi_{s}(x) d s+\sum_{k=1}^{m} \nabla a_{k}\left(\varphi_{s}(x)\right) \nabla \varphi_{s}(x) d w_{k}(s)
$$

on the interval $s \in\left[t, \tau_{t}(x)\right)$, since the usual stochastic differential equation without reflection holds for $s \in\left[t, \tau_{t}(x)\right)$. For $t$ belonging to the set

$$
A(x)=\left\{t \geq 0: \varphi_{t}(x) \in \mathbb{R}^{d-1} \times\{0\}\right\}
$$

where the solution reaches the hyperplane $\mathbb{R}^{d-1} \times\{0\}$, we set

$$
\nabla \varphi_{t}(x)=P \nabla \varphi_{t}(x) \text {. }
$$

Here $P$ is the linear operator that reduces to zero the last row of a $d \times d$ matrix. This can be heuristically explained by the fact that the coordinate $d$ of the mapping $\varphi_{t}(\cdot)$ attains its minimum at the point $x$.

A formal definition of equations of this kind and a theorem on the existence and uniqueness of the solution are given in Section 2. We establish the equation for $\nabla \varphi_{t}$ in Section 3 .

\section{The SOlution of A StOchastic EQUATion}

Let $w_{1}(t), \ldots, w_{m}(t)$ be independent Wiener processes,

$$
\mathcal{F}_{t}=\sigma\left(w_{k}(s), k=1, \ldots, m, s \leq t\right),
$$

$x_{t}$ a continuous $\mathcal{F}_{t}$-adapted stochastic process, and

$$
a_{k}: \mathbb{R}^{l} \times \mathbb{R}^{m} \rightarrow \mathbb{R}^{l}, \quad b_{k}: \mathbb{R}^{l} \times \mathbb{R}^{m} \rightarrow \mathbb{R}^{m} .
$$

Consider the measure-valued stochastic process $\nu(t)=\delta_{0} \mathbb{1}_{\{x(t)=0\}}$, where $\delta_{0}$ is the probability measure assigning the unit mass to zero.

Definition 2.1. We say that a pair of $\mathcal{F}_{t}$-measurable stochastic processes $\left(y_{t}, z_{t}\right)$ satisfies the system

$$
\left\{\begin{array}{l}
d y_{t}=a_{0}\left(y_{t}, z_{t}\right) d t+\sum_{k=1}^{m} a_{k}\left(y_{t}, z_{t}\right) d w_{k}(t)-y_{t-} \nu(d t) \\
d z_{t}=b_{0}\left(y_{t}, z_{t}\right) d t+\sum_{k=1}^{m} b_{k}\left(y_{t}, z_{t}\right) d w_{k}(t), t \geq 0
\end{array}\right.
$$

if

1) $y_{t}, t \geq 0$, is a càdlàg process;

2) $z_{t}, t \geq 0$, has continuous trajectories; 
3) $z_{t}=z_{0}+\int_{0}^{t} b_{0}\left(y_{s}, z_{s}\right) d s+\sum_{k=1}^{m} \int_{0}^{t} b_{k}\left(y_{s}, z_{s}\right) d w_{k}(s), t \geq 0$ almost surely;

4) for almost all $\omega$, the set $\left\{t \geq 0: x_{t}=0\right\}$ belongs to $\left\{t \geq 0: y_{t}=0\right\}$;

5) for an arbitrary stopping time $\tau$ such that $x_{\tau} \neq 0$, we have

$$
y_{t}=y_{\tau}+\int_{\tau}^{t} a_{0}\left(y_{s}, z_{s}\right) d s+\sum_{k=1}^{m} \int_{\tau}^{t} a_{k}\left(y_{s}, z_{s}\right) d w_{k}(s)
$$

with probability one for all $t \in\left[\tau, \tau^{\circ}\right)$ where $\tau^{\circ}=\inf \left\{t \geq \tau: x_{t}=0\right\}$.

Remark 2.1. If $x_{t} \neq 0$ for all $t \geq 0$, then $\left(y_{t}, z_{t}\right)$ is a solution of the system of ordinary stochastic differential equations

$$
\begin{aligned}
& d y_{t}=a_{0}\left(y_{t}, z_{t}\right) d t+\sum_{k=1}^{m} a_{k}\left(y_{t}, z_{t}\right) d w_{k}(t), \\
& d z_{t}=b_{0}\left(y_{t}, z_{t}\right) d t+\sum_{k=1}^{m} b_{k}\left(y_{t}, z_{t}\right) d w_{k}(t) .
\end{aligned}
$$

Remark 2.2. Assume that there exists a finite family of stopping times

$$
0<\tau_{1}<\tau_{2}<\cdots<\tau_{n}
$$

such that the process $x_{t}$ equals zero at these and only these points.

Then the solution $\left(y_{t}, z_{t}\right)$ of system $(2)$ with initial conditions $\left(y_{0}, z_{0}\right)$ can be determined by using a recurrence.

We take $\left(y_{t}, z_{t}\right)$ for $t \in\left[0, \tau_{1}\right)$ as a solution of $(3)$ with the initial condition $\left(y_{0}, z_{0}\right)$. On the intervals $\left[\tau_{k}, \tau_{k+1}\right)$, we use the solution of (3) with the initial condition

$$
z_{\tau_{k}}:=z_{\tau_{k}-}, \quad y_{\tau_{k}}:=0 .
$$

Finding a solution of (2) may be difficult if the set

$$
\left\{t \geq 0: x_{t}=0\right\}
$$

is uncountable. This is the case, for example, if $x_{t}$ is a Wiener process.

Theorem 2.1. Assume that functions $a_{k}$ and $b_{k}, k=0, \ldots, m$, are Lipschitz continuous; that is, there exists a constant $L>0$ such that

$$
\left|a_{k}\left(y_{1}, z_{1}\right)-a_{k}\left(y_{2}, z_{2}\right)\right|+\left|b_{k}\left(y_{1}, z_{1}\right)-b_{k}\left(y_{2}, z_{2}\right)\right| \leq L\left(\left|y_{1}-y_{2}\right|+\left|z_{1}-z_{2}\right|\right)
$$

for all $k$ and for all $y_{1}, y_{2}, z_{1}$, and $z_{2}$.

Then (2) has a unique solution for all nonrandom initial conditions $\left(y_{0}, z_{0}\right)$.

Proof. Assume that $\left(y_{t}, z_{t}\right)$ is a solution of (2). We estimate the moments of $y_{t}$ and $z_{t}$.

Let $c>0$ and $N>0$. Put

$$
\begin{aligned}
\sigma_{0}(c) & = \begin{cases}0, & x_{0} \neq 0, \\
\inf \left\{t \geq 0:\left|x_{t}\right|=c\right\}, & x_{0}=0,\end{cases} \\
\tau_{0}(c) & =\inf \left\{t \geq 0: x_{t}=0\right\}, \\
\sigma_{j}(c) & =\inf \left\{t \geq \tau_{j}(c):\left|x_{t}\right|=c\right\}, \\
\tau_{j+1}(c) & =\inf \left\{t \geq \sigma_{j}(c):\left|x_{t}\right|=0\right\}, \\
\theta_{N} & =\inf \left\{t \geq 0:\left|z_{t}\right|+\left|y_{t}\right| \geq N\right\} .
\end{aligned}
$$

In what follows we use several constants $K_{1}, K_{2}, \ldots$ For simplicity, we write $K$. for all of them, though they may have different values.

The Itô formula and Burkholder inequality imply that

$$
\mathrm{E} \sup _{s \in[0, t]}\left|z_{s \wedge \theta_{N}}\right|^{2} \leq K .\left(1+\int_{0}^{t} \mathrm{E}\left(\left|z_{s \wedge \theta_{N}}\right|^{2}+\left|y_{s \wedge \theta_{N}}\right|^{2}\right)\right) d s, \quad t \in[0, T] .
$$


Let $c>0$. Assume that

$$
t \in \bigcup_{j}\left[\sigma_{j}(c), \tau_{j+1}(c)\right)
$$

Then Definition 2.1 implies that

$$
y_{t}=\sum_{j \geq 1}\left(y_{\sigma_{j}(c)}+\int_{\sigma_{j}(c)}^{t} a_{0}\left(y_{s}, z_{s}\right) d s+\sum_{k=1}^{m} \int_{\sigma_{j}(c)}^{t} a_{k}\left(y_{s}, z_{s}\right) d w_{k}(s)\right) \mathbb{1}_{t \in\left[\sigma_{j}(c), \tau_{j+1}(c)\right)} .
$$

Note that if $x_{t} \neq 0$, then there exists $c_{0}=c_{0}(\omega)$ such that

$$
t \in \bigcap_{0<c<c_{0}} \bigcup_{j \geq 1}\left[\sigma_{j}(c), \tau_{j+1}(c)\right)
$$

Let $j_{c}$ be a number such that $t \in\left[\sigma_{j_{c}}(c), \tau_{j_{c}+1}(c)\right)$. It is easy to see that the limit

$$
\sigma:=\lim _{c \rightarrow 0+} \sigma_{j_{c}}(c)
$$

exists in this case. Moreover, Definition 2.1 implies that the following limits

$$
\begin{gathered}
\lim _{c \rightarrow 0+} y_{\sigma_{j_{c}}(c)}= \begin{cases}0, & \sigma \neq 0, \\
y_{0}, & \sigma=0,\end{cases} \\
\lim _{c \rightarrow 0+} \int_{\sigma_{j_{c}}(c)}^{t} a_{0}\left(y_{s}, z_{s}\right) d s=\int_{\sigma}^{t} a_{0}\left(y_{s}, z_{s}\right) d s
\end{gathered}
$$

exist, too.

Since $y_{t}=0$ for $x_{t}=0$, we have that for all $t \in[0, T]$

$$
\begin{aligned}
\left|y_{t}\right| \leq & \frac{\lim }{c \downarrow 0} \sum_{j \geq 1}\left(\mid y_{\sigma_{j}(c)}+\int_{\sigma_{j}(c)}^{t} a_{0}\left(y_{s}, z_{s}\right) d s\right. \\
& \left.\quad+\sum_{k=1}^{m} \int_{\sigma_{j}(c)}^{t} a_{k}\left(y_{s}, z_{s}\right) d w_{k}(s) \mid\right) \mathbb{1}_{t \in\left[\sigma_{j}(c), \tau_{j+1}(c)\right)} \\
\leq & \left|y_{0}\right|+\lim _{c \downarrow 0} \sum_{j \geq 1}\left|\int_{\sigma_{j}(c)}^{t} a_{0}\left(y_{s}, z_{s}\right) d s\right| \mathbb{1}_{t \in\left[\sigma_{j}(c), \tau_{j+1}(c)\right)} \\
& +\sum_{k=1}^{m} \frac{\lim }{c \downarrow 0} \sum_{j \geq 1} \int_{\sigma_{j}(c)}^{t} a_{k}\left(y_{s}, z_{s}\right) d w_{k}(s) \mathbb{1}_{t \in\left[\sigma_{j}(c), \tau_{j+1}(c)\right)} \\
\leq & \left|y_{0}\right|+\int_{0}^{t}\left|a_{0}\left(y_{s}, z_{s}\right)\right| d s \\
& +\frac{\lim }{c \downarrow 0} \sum_{j \geq 1} \sum_{k=1}^{m}\left|\int_{\sigma_{j}(c) \wedge t}^{\tau_{j+1}(c) \wedge t} a_{k}\left(y_{s}, z_{s}\right) d w_{k}(s)\right| \mathbb{1}_{t \in\left(\sigma_{j}(c), \tau_{j+1}(c)\right) .}
\end{aligned}
$$


The Burkholder inequality and Fatou lemma imply

$$
\begin{aligned}
& \mathrm{E} \sup _{s \in[0, t]}\left|y_{s \wedge \theta_{N}}\right|^{2} \leq K .\left(1+\int_{0}^{t} \mathrm{E}\left(\left|z_{s \wedge \theta_{N}}\right|^{2}+\left|y_{s \wedge \theta_{N}}\right|^{2}\right) d s\right. \\
& \left.\quad+\frac{\lim _{c \downarrow 0}}{j \geq 1} \sum_{k=1}^{m} \mathrm{E} \sup _{s \in[0, t]}\left|\int_{\sigma_{j}(c) \wedge s \wedge \theta_{N}}^{\tau_{j+1}(c) \wedge s \wedge \theta_{N}} a_{k}\left(y_{r}, z_{r}\right) d w_{k}(r)\right|^{2}\right) \\
& \leq K \cdot\left(1+\int_{0}^{t} \mathrm{E}\left(\left|z_{s \wedge \theta_{N}}\right|^{2}+\left|y_{s \wedge \theta_{N}}\right|^{2}\right) d s\right. \\
& \left.\quad+\frac{\lim _{c \downarrow 0}}{\sum_{j \geq 1}} \int_{0}^{t} \mathrm{E} \mathbb{1}_{\left[\sigma_{j}(c), \tau_{j+1}(c)\right]}(s)\left(1+\left|y_{s \wedge \theta_{N}}\right|^{2}+\left|z_{s \wedge \theta_{N}}\right|^{2}\right) d s\right) \\
& \leq K .\left(1+\int_{0}^{t}\left(\left|y_{s \wedge \theta_{N}}\right|^{2}+\left|z_{s \wedge \theta_{N}}\right|^{2}\right) d s\right), \quad t \in[0, T] .
\end{aligned}
$$

Applying the Gronwall lemma to (6) and (8), we get the bound

$$
\mathrm{E} \sup _{s \in[0, T]}\left(\left|y_{s \wedge \theta_{N}}\right|^{2}+\left|z_{s \wedge \theta_{N}}\right|^{2}\right) \leq K<\infty
$$

where $K$ does not depend on $N$. Letting $N \rightarrow \infty$ we prove that

$$
\mathrm{E} \sup _{s \in[0, T]}\left(\left|y_{s}\right|^{2}+\left|z_{s}\right|^{2}\right) \leq K<\infty .
$$

Remark 2.3. One can check in a similar way that

$$
\mathrm{E} \sup _{t \in[0, T]}\left(\left|y_{t}\right|^{p}+\left|z_{t}\right|^{p}\right)<\infty
$$

for all $p>1$.

Uniqueness. Assume that $\left(y_{t}, z_{t}\right)$ and $\left(\widetilde{y}_{t}, \widetilde{z}_{t}\right)$ are solutions of (2). Similarly to the proof of (6) and (8) we obtain

$$
\begin{aligned}
& \mathrm{E} \sup _{s \in[0, T]}\left(\left|y_{s}-\widetilde{y}_{s}\right|^{2}+\left|z_{s}-\widetilde{z}_{s}\right|^{2}\right) \\
& \leq K .\left(\mathrm{E} \int_{0}^{t}\left(\left|y_{s}-\widetilde{y}_{s}\right|^{2}+\left|z_{s}-\widetilde{z}_{s}\right|^{2}\right) d s\right. \\
& \left.\quad+\frac{\lim _{c \downarrow 0}}{\leq} \sum_{j \geq 1}^{m} \mathrm{E} \sup _{s \in I_{j}(c)}\left|\int_{\sigma_{j}(c) \wedge t \wedge s}^{\tau_{j+1}(c) \wedge t \wedge s}\left(a_{k}\left(y_{r}, z_{r}\right)-a_{k}\left(\widetilde{y}_{r}, \widetilde{z}_{r}\right)\right) d w_{k}(r)\right|^{2}\right) \\
& \leq K . \mathrm{E} \int_{0}^{t}\left(\left|y_{s}-\widetilde{y}_{s}\right|^{2}+\left|z_{s}-\widetilde{z}_{s}\right|^{2}\right) d s .
\end{aligned}
$$

Here

$$
I_{j}(c)=\left[\sigma_{j}(c), \tau_{j+1}(c)\right] .
$$

Now the Gronwall lemma implies that $y_{t}=\widetilde{y}_{t}$ and $z_{t}=\widetilde{z}_{t}$ almost surely. 
Existence. Let $c>0$. Define the processes $y_{t}^{c}$ and $z_{t}^{c}$ as follows:

$$
\begin{gathered}
y_{0}^{c}:=y_{0}, \quad z_{0}^{c}:=z_{0}, \\
\left\{\begin{aligned}
& y_{t}^{c}=y_{\sigma_{j}(c)}^{c}+\int_{\sigma_{j}(c)}^{t} a_{0}\left(y_{s}^{c}, z_{s}^{c}\right) d s \text { for } t \in\left[\sigma_{j}(c), \tau_{j+1}(c)\right), \\
&+\sum_{k=1}^{m} \int_{\sigma_{j}(c)}^{t} a_{k}\left(y_{s}^{c}, z_{s}^{c}\right) d w_{k}(s) \text { for } t \in\left[\tau_{j}(c), \sigma_{j}(c)\right], \\
& y_{t}^{c}=0 \quad t \in[0, T] .
\end{aligned}\right. \\
z_{t}^{c}=z_{0}+\int_{0}^{t} b_{0}\left(y_{s}^{c}, z_{s}^{c}\right) d s+\sum_{k=1}^{m} b_{k}\left(y_{s}^{c}, z_{s}^{c}\right) d w_{k}(s), \quad t \in
\end{gathered}
$$

Since $x_{t}$ is a continuous process, the total number of intervals $\left[\sigma_{j}(c), \tau_{j+1}(c)\right) \subset[0, T]$ is finite for all $c>0$. The pair of processes $\left(y_{t}^{c}, z_{t}^{c}\right)$ is a solution of a system of stochastic differential equations with Lipschitz coefficients on the interval $\left[\sigma_{j}(c), \tau_{j+1}(c)\right)$. Thus these processes are uniquely determined. Similarly, $y^{c} \equiv 0$ and $z_{t}^{c}$ are uniquely determined from equation (12) on the interval $\left[\tau_{j}(c), \sigma_{j}(c)\right]$.

The bound

$$
\sup _{c>0} \mathrm{E} \sup _{t \in[0, T]}\left(\left|y_{s}^{c}\right|^{p}+\left|z_{s}^{c}\right|^{p}\right)<\infty
$$

for all $p>1$ can be obtained similarly to the proof of (9). Put

$$
U\left(c_{1}, c_{2}\right)=\left(\bigcup_{j \geq 1}\left[\sigma_{j}\left(c_{1}\right), \tau_{j+1}\left(c_{1}\right)\right)\right) \triangle \bigcup_{j \geq 1}\left[\sigma_{j}\left(c_{2}\right), \tau_{j+1}\left(c_{2}\right)\right) .
$$

Then (see (10))

$$
\begin{aligned}
& \mathrm{E} \sup _{s \in[0, t]}\left(\left|y_{s}^{c_{1}}-y_{s}^{c_{2}}\right|^{2}+\left|z_{s}^{c_{1}}-z_{s}^{c_{2}}\right|^{2}\right) \\
& \leq K . \mathrm{E} \int_{0}^{t}\left(\left|y_{s}^{c_{1}}-y_{s}^{c_{2}}\right|^{2}+\left|z_{s}^{c_{1}}-z_{s}^{c_{2}}\right|^{2}\right) d s \\
&+K . \mathrm{E} \int_{0}^{t} \mathbb{1}_{U\left(c_{1}, c_{2}\right)}(s)\left(1+\left|y_{s}^{c_{1}}\right|^{2}+\left|y_{s}^{c_{2}}\right|^{2}+\left|z_{s}^{c_{1}}\right|^{2}+\left|z_{s}^{c_{2}}\right|^{2}\right) d s \\
& \leq K . \mathrm{E} \int_{0}^{t}\left(\left|y_{s}^{c_{1}}-y_{s}^{c_{2}}\right|^{2}+\left|z_{s}^{c_{1}}-z_{s}^{c_{2}}\right|^{2}\right) d s \\
&+K \cdot\left(\mathrm{E} \int_{0}^{T} \mathbb{1}_{U\left(c_{1}, c_{2}\right)}(s) d s\right)^{1 / 2}, \quad t \in[0, T] .
\end{aligned}
$$

Note that $\bigcup_{j \geq 1}\left[\sigma_{j}(c), \tau_{j+1}(c)\right) \subset\left\{t \in[0, T]: x_{t} \neq 0\right\}$ and

$$
\left\{t \in[0, T]: x_{t} \neq 0\right\}=\bigcup_{C>0} \bigcap_{c \in(0, C)} \bigcup_{j \geq 1}\left[\sigma_{j}(c), \tau_{j+1}(c)\right) .
$$

Thus

$$
\lambda\left(\bigcup_{j}\left[\tau_{j}\left(c_{1}\right), \sigma_{j}\left(c_{1}\right)\right]\right) \triangle\left(\bigcup_{j}\left[\tau_{j}\left(c_{2}\right), \sigma_{j}\left(c_{2}\right)\right]\right) \rightarrow 0, \quad c_{1}, c_{2} \rightarrow 0+.
$$

Hence the second term approaches zero as $c_{1}, c_{2} \rightarrow 0+$ by the Lebesgue dominated convergence theorem. Thus the left hand side of (13) also approaches zero by the Gronwall lemma. Therefore the sequence of processes $y^{c}$. and $z^{c}$. converges in $L_{2}$ as $c \rightarrow 0+$. It is easy to check that the limit process satisfies (2). Theorem 2.1 is proved. 


\section{The Equation For the Derivative $\partial \varphi_{t}(x) / \partial x$}

Let $\varphi_{t}(x)$ be a solution of (1). Consider the approximating sequence of processes determined by the following system of stochastic differential equations:

$$
\left\{\begin{aligned}
d \varphi_{t}^{(\varepsilon)}(x)= & \left(a_{0}\left(\varphi_{t}^{(\varepsilon)}(x)\right)+g_{\varepsilon}\left(\varphi_{t}^{(\varepsilon)}(x)\right)\right) d t \\
& +\sum_{k=1}^{m} a_{k}\left(\varphi_{t}^{(\varepsilon)}(x)\right) d w_{k}(t), t \geq 0 \\
\varphi_{0}^{(\varepsilon)}(x)= & x, x \in \mathbb{R}^{d-1} \times(0, \infty)
\end{aligned}\right.
$$

where $g_{\varepsilon}(x)=\left(0, \ldots, 0, \psi\left(x_{d} / \varepsilon\right) / x_{d}^{2}\right), \psi \in C^{\infty}((0, \infty)), \psi(x)=0$ for $x \geq 2$ and $\psi(x)=1$ for $x \leq 1$, and $\psi$ is nonincreasing.

It is proved in the paper [2] that a solution of (14) exists for all $x \in \mathbb{R}^{d-1} \times(0, \infty)$ and $t \geq 0$ and belongs to the half-space $\mathbb{R}^{d-1} \times(0, \infty)$. Moreover

1) there exists a continuous version of $\varphi_{t}^{(\varepsilon)}(x)$ with respect to the pair of variables $(t, x)$

2) for all $T>0, p>1$, and $r>0$

$$
\begin{aligned}
& \sup _{\varepsilon \in(0,1]} \sup _{\substack{|x| \leq r \\
x \in \mathbb{R}^{d-1} \times(0, \infty)}} \mathrm{E} \sup _{t \in[0, T]}\left(\left|\varphi_{t}^{(\varepsilon)}(x)\right|^{p}+\left|\varphi_{t}(x)\right|^{p}\right)<\infty, \\
& \sup _{\substack{|x| \leq r \\
x \in \mathbb{R}^{d-1} \times(0, \infty)}} \mathrm{E} \sup _{t \in[0, T]}\left|\varphi_{t}^{(\varepsilon)}(x)-\varphi_{t}(x)\right|^{p} \rightarrow 0, \quad \varepsilon \rightarrow 0+.
\end{aligned}
$$

Let $y_{t}^{(\varepsilon)}(x)$ be a solution of the following linear stochastic differential equation

$$
\left\{\begin{aligned}
d y_{t}^{(\varepsilon)}(x)= & \left(\nabla a_{0}\left(\varphi_{t}^{(\varepsilon)}(x)\right)+\nabla g_{\varepsilon}\left(\varphi_{t}^{(\varepsilon)}(x)\right)\right) y_{t}^{(\varepsilon)}(x) d t \\
& +\sum_{k=1}^{m} \nabla a_{k}\left(\varphi_{t}^{(\varepsilon)}(x)\right) y_{t}^{(\varepsilon)}(x) d w_{k}(t), \quad t \geq 0 \\
y_{0}^{(\varepsilon)}(x)=\mathbb{1}, & x \in \mathbb{R}^{d-1} \times(0, \infty),
\end{aligned}\right.
$$

where $\mathbb{1}$ is the unit $d \times d$ matrix.

Denote by $n(x, d t)$ a point random measure such that $n(x,\{t\})=1$ if and only if $\varphi_{t}(x)$ belongs to the hyperspace $\mathbb{R}^{d-1} \times\{0\}$.

Consider the matrix $P=\left(p_{i j}\right)_{i, j=1}^{d}$ defined as follows: $p_{i j}=1$ for $i=j=d$ and $p_{i j}=0$ otherwise.

The following assertion plays a key role in what follows for establishing an equation: for the derivative $\nabla \varphi_{t}(x)$.

Theorem 3.1. Assume that the functions $a_{k}: \mathbb{R}_{+}^{d} \rightarrow \mathbb{R}^{d}, k=0, \ldots, m$, are continuously differentiable and have bounded partial derivatives.

Assume that

$$
\sum_{k=1}^{m}\left(a_{k, d}(x)\right)^{2}>0 \quad \text { for all } x \in \mathbb{R}^{d-1} \times\{0\},
$$

where $a_{k, d}$ is the $d$ th coordinate of the function $a_{k}=\left(a_{k, 1}, \ldots, a_{k, d}\right)^{T}$.

Then

$$
\mathrm{E}\left|y_{t}^{(\varepsilon)}(x)-y_{t}(x)\right|^{p} \rightarrow 0, \quad \varepsilon \rightarrow 0+,
$$


for all $t \in[0, T], x \in \mathbb{R}^{d-1} \times(0, \infty)$, and $p>1$ where $y_{t}(x), t \geq 0$, is a solution of the equation

$$
\left\{\begin{aligned}
d y_{t}(x)= & \nabla a_{0}\left(\varphi_{t}(x)\right) y_{t}(x) d t \\
& +\sum_{k=1}^{m} \nabla a_{k}\left(\varphi_{t}(x)\right) y_{t}(x) d w_{k}(t)-P y_{t-}(x) n(x, d t), \\
y_{0}(x)= & \mathbb{1},
\end{aligned}\right.
$$

for all $t \in[0, T], x \in \mathbb{R}^{d-1} \times(0, \infty)$, and $p>1$.

Remark 3.1. Equation (20) is understood in the sense of Section 1. The $d$ th coordinate of the process $\varphi_{t}(x)$ is considered as the process $x_{t}$, the $d$ th row of the process $y_{t}(x)$, as $y_{t}$, and the first $(d-1)$ rows of the process $y_{t}(x)$ combined with $\varphi_{t}(x)$, as $z_{t}$.

Remark 3.2. Let $x \in \mathbb{R}_{+}^{d}$. The trajectories of the process $y_{t}^{(\varepsilon)}(x), t \in[0, T]$, are continuous with respect to $t$, while those of $y_{t}(x), t \in[0, T]$, are not continuous in general. Thus the processes $y_{t}^{(\varepsilon)}(x)$ cannot converge (even in the weak sense) in the Skorokhod topology to $y_{t}(x)$ in the space $D_{[0, T]}$ of right continuous functions that have left limits (see [5]). On the other hand, one can check that $y_{t}^{(\varepsilon)}(x), t \in[0, T]$, converge to $y_{t}(x), t \in[0, T]$, in the space $D_{[0, T]}$ equipped with the topology $S$ (see [7]).

Proof. To prove Theorem 3.1 we need the following auxiliary result.

Lemma 3.1. For all $p>1$, we have

$$
\begin{gathered}
\sup _{\varepsilon \in(0,1]} \sup _{x \in \mathbb{R}^{d-1} \times(0, \infty)} \mathrm{E} \sup _{t \in[0, T]}\left|y_{t}^{(\varepsilon)}(x)\right|^{p}<\infty, \\
\sup _{x \in \mathbb{R}^{d-1} \times(0, \infty)} \mathrm{E} \sup _{t \in[0, T]}\left|y_{t}(x)\right|^{p}<\infty .
\end{gathered}
$$

The proof of (21) is standard. The proof of (22) is similar to that of (9).

It view of (21) convergence (19) follows from convergence in probability,

$$
y_{t}^{(\varepsilon)}(x) \stackrel{\mathrm{P}}{\rightarrow} y_{t}(x), \quad \varepsilon \rightarrow 0+.
$$

Thus we restrict ourselves to the case of $p=2$ in the proof of Theorem 3.1.

Let $l \in\{1, \ldots, d\}$ and $x \in \mathbb{R}^{d-1} \times(0, \infty)$ be fixed. Denote the $l$ th columns of the matrices $y_{t}^{(\varepsilon)}(x)$ and $y_{t}(x)$ by $y^{(\varepsilon)}(t)$ and $y(t)$, respectively. Put $\varphi(t)=\varphi_{t}(x)$,

$$
\bar{y}^{(\varepsilon)}(t)=\left(y_{1}^{(\varepsilon)}(t), \ldots, y_{d-1}^{(\varepsilon)}(t)\right)^{T},
$$

and $\xi(t)=\xi(x, t)$. Note that $y^{(\varepsilon)}(t)$ and $y(t)$ satisfy an equation of the same form as in the case of $y_{t}^{(\varepsilon)}(x)$ and $y_{t}(x)$ but with the following initial conditions:

$$
y^{(\varepsilon)}(0)=y(0)=(0, \ldots, 1, \ldots, 0)^{T}
$$

(all coordinates of this vector are zero except for the $l$ th coordinate).

We prove the convergence

$$
y^{(\varepsilon)}(t) \stackrel{L_{2}}{\rightarrow} y(t), \quad \varepsilon \rightarrow 0+.
$$

Note that

$$
\begin{aligned}
\mathrm{E}\left|\bar{y}^{(\varepsilon)}(t)-\bar{y}(t)\right|^{2} \leq & K . \mathrm{E} \int_{0}^{t} \sum_{k=0}^{m}\left|\nabla a_{k}\left(\varphi^{(\varepsilon)}(s)\right)-\nabla a_{k}(\varphi(s))\right|^{2}|y(s)|^{2} d s \\
& +K . \mathrm{E} \int_{0}^{t}\left(\left|\bar{y}^{(\varepsilon)}(s)-\bar{y}(s)\right|^{2}+\left|y_{d}^{(\varepsilon)}(s)-y_{d}(s)\right|^{2}\right) d s
\end{aligned}
$$


The first term in (23) converges to zero as $\varepsilon \rightarrow 0+$ by (15), (9), and the Lebesgue dominated convergence theorem. Applying the Gronwall lemma to (23), we complete the proof of the theorem if we verify that

$$
\begin{gathered}
\mathrm{E}\left(y_{d}^{(\varepsilon)}(t)-y_{d}(t)\right)^{2} \rightarrow 0, \quad \varepsilon \rightarrow 0+, \\
\int_{0}^{T} \mathrm{E}\left(y_{d}^{(\varepsilon)}(s)-y_{d}(s)\right)^{2} d s \rightarrow 0, \quad \varepsilon \rightarrow 0+.
\end{gathered}
$$

Let $c>0$. Consider the stopping times

$$
\begin{gathered}
\sigma_{0}(c)=0, \\
\bar{\sigma}_{n}(b)=\inf \left\{t \geq \sigma_{n}(c): \varphi_{d}(t) \leq b\right\}, \quad b \in[0, c), \\
\sigma_{n+1}(c)=\inf \left\{t \geq \bar{\sigma}_{n}(0): \varphi_{d}(t)=c\right\} .
\end{gathered}
$$

In other words, $\bar{\sigma}_{n}(b)$ is the first stopping time after $\sigma_{n}(c)$ when the process $\varphi_{d}(t)$ reaches the level $b$, while $\sigma_{n+1}(c)$ is the first moment after $\bar{\sigma}_{n}(b)$ when the process $\varphi_{d}(t)$ reaches the level $c$ after its visit of the origin.

Note that if $b>3 \varepsilon$, then $g_{\varepsilon}\left(\varphi^{(\varepsilon)}(s)\right)=0$ for $s \in \bigcup_{n}\left[\sigma_{n}(c) ; \bar{\sigma}_{n}(b)\right]$. Therefore

$$
\begin{aligned}
y_{d}^{(\varepsilon)}(s)= & \sum_{n \geq 0} \mathbb{1}_{Q^{\prime}(\varepsilon, s, n)}\left(y_{d}^{(\varepsilon)}\left(\sigma_{n}(c)\right)\right. \\
& +\int_{\sigma_{m}(c) \wedge s}^{\bar{\sigma}_{m}(b) \wedge s} a_{0, d}^{\prime}\left(\varphi^{(\varepsilon)}(z)\right) y^{(\varepsilon)}(z) d z \\
& \left.+\sum_{k=1}^{m} \int_{\sigma_{n}(c) \wedge s}^{\bar{\sigma}_{n}(b) \wedge s} a_{k, d}^{\prime}\left(\varphi^{(\varepsilon)}(z)\right) y^{(\varepsilon)}(z) d w_{k}(z)\right) \\
& +y_{d}^{(\varepsilon)}(s) \mathbb{1}_{Q^{\prime \prime}(\varepsilon, s)},
\end{aligned}
$$

where $a_{k, j}^{\prime}$ is the gradient of the $j$ th coordinate of the function $a_{k}$ and

$$
\begin{gathered}
Q^{\prime}(\varepsilon, s, n)=\left\{s \in\left[\sigma_{n}(c), \bar{\sigma}_{n}(b)\right]\right\} \cap\left\{\sup _{t \in[0, T]}\left|\varphi^{(\varepsilon)}(t)-\varphi(t)\right|<b / 3\right\}, \\
Q^{\prime \prime}(\varepsilon, s)=\left\{s \notin \bigcup_{n \geq 0}\left[\sigma_{n}(c) ; \bar{\sigma}_{n}(b)\right]\right\} \cup\left\{\sup _{t \in[0, T]}\left|\varphi^{(\varepsilon)}(t)-\varphi(t)\right| \geq b / 3\right\} .
\end{gathered}
$$

Thus

$$
\begin{array}{r}
\mathrm{E}\left|y_{d}^{(\varepsilon)}(s)-y_{d}(s)\right|^{2} \\
\leq K \cdot\left(\sum _ { n \geq 0 } \mathrm { E } \left(\mathbb{1}_{Q^{\prime}(\varepsilon, s, n)}\left|y_{d}^{(\varepsilon)}\left(\sigma_{n}(c)\right)-y_{d}\left(\sigma_{n}(c)\right)\right|^{2}\right.\right. \\
+\sum_{k=0}^{m} \int_{\sigma_{n}(c) \wedge s}^{\bar{\sigma}_{n}(b) \wedge s}\left|a_{k, d}^{\prime}\left(\varphi^{(\varepsilon)}(z)\right)-a_{k, d}^{\prime}(\varphi(z))\right|^{2}|y(z)|^{2} d z \\
\left.+\sum_{k=0}^{m} \int_{\sigma_{n}(c) \wedge s}^{\bar{\sigma}_{n}(b) \wedge s}\left|a_{k, d}^{\prime}\left(\varphi^{(\varepsilon)}(z)\right)\right|^{2}\left|y^{(\varepsilon)}(z)-y(z)\right|^{2} d z\right) \\
\left.+\mathrm{E} \sup _{z \in[0, T]}\left(\left|y_{d}^{(\varepsilon)}(z)\right|^{2}+\left|y_{d}(z)\right|^{2}\right) \mathbb{1}_{Q^{\prime \prime}(\varepsilon, s)}\right)
\end{array}
$$




$$
\begin{aligned}
\leq K . & \left(\sum_{n \geq 0} \mathrm{E} \mathbb{1}_{\left\{s \in\left[\sigma_{n}(c), \bar{\sigma}_{n}(b)\right]\right\}}\left|y_{d}^{(\varepsilon)}\left(\sigma_{n}(c)\right)-y_{d}\left(\sigma_{n}(c)\right)\right|^{2}\right. \\
& +\int_{0}^{T} \sum_{k=0}^{m} \sqrt{\mathrm{E}\left|a_{k, d}^{\prime}\left(\varphi^{(\varepsilon)}(z)\right)-a_{k, d}^{\prime}(\varphi(z))\right|^{4}} d z \\
& +\int_{0}^{s} \mathrm{E}\left|y^{(\varepsilon)}(z)-y(z)\right|^{2} d z \\
& \left.+\sqrt{\mathrm{P}\left(\left\{s \notin \bigcup_{n \geq 0}\left[\sigma_{n}(c) ; \bar{\sigma}_{n}(b)\right]\right\} \cup\left\{\sup _{t \in[0, T]}\left|\varphi^{(\varepsilon)}(s)-\varphi(s)\right| \geq b / 3\right\}\right)}\right)
\end{aligned}
$$

for all $s \in[0, T]$ (see (7) and (8)). We used the Cauchy inequality, (9), and Lemma 3.1 in the proof of the last inequality.

Consider the set

$$
\begin{aligned}
A_{\varepsilon, r, b, c, \delta, \eta, \varkappa}= & \left\{\sup _{z \in[0, T]}\left(\left|y^{(\varepsilon)}(z)\right|+|y(z)|\right)>r\right\} \\
& \cup\left\{\sup _{z \in[0, T]}\left|\varphi^{(\varepsilon)}(z)-\varphi(z)\right|>\frac{b}{3}\right\} \\
& \cup\left\{\lambda\left([0, T] \backslash \bigcup_{n}\left[\sigma_{n}(c), \bar{\sigma}_{n}(b)\right]\right)>\eta\right\} \\
& \cup\left\{\exists s_{1}, s_{2} \in[0, T],\left|s_{1}-s_{2}\right|<\eta:\right. \\
& \sum_{k=1}^{m}\left(\left|\int_{s_{1}}^{s_{2}} a_{k, d}^{\prime}\left(\varphi^{(\varepsilon)}(z)\right) d w_{k}(z)\right|+\left|\int_{s_{1}}^{s_{2}} a_{k, d}^{\prime}(\varphi(z)) d w_{k}(z)\right|\right) \\
& \left.+\left|\int_{s_{1}}^{s_{2}} a_{0, d}^{\prime}\left(\varphi^{(\varepsilon)}(z)\right) y^{(\varepsilon)}(z) d z\right|+\left|\int_{s_{1}}^{s_{2}} a_{0, d}^{\prime}(\varphi(z)) y(z) d z\right|>\delta\right\} \\
& \cup\left\{\exists n: \xi_{\varepsilon}\left(\sigma_{n}(c)\right)-\xi_{\varepsilon}\left(\bar{\sigma}_{n}(b)\right) \leq \varkappa\right\} \\
& \cup\left\{\exists n:\left|y^{d}\left(\sigma_{n}(c)\right)\right|>\delta\right\},
\end{aligned}
$$

where $\lambda$ is Lebesgue measure on $\mathbb{R}^{1}$,

$$
\xi_{\varepsilon}(t)=\int_{0}^{t}\left(g_{\varepsilon}\left(\varphi^{(\varepsilon)}(s)\right), \bar{n}\right) d s, \quad \bar{n}=(0, \ldots, 0,1) .
$$

It is easy to deduce from (16) that for all $p>1$,

$$
\mathrm{E} \sup _{t \in[0, T]}\left|\xi_{\varepsilon}(t)-\xi(t)\right|^{p} \rightarrow 0 . \quad \varepsilon \rightarrow 0+.
$$


The second term on the right hand side of (24) approaches zero as $\varepsilon \rightarrow 0+$ by the Lebesgue dominated convergence theorem. Then (24) implies

$$
\begin{aligned}
\int_{0}^{t} \mathrm{E}\left(y_{d}^{(\varepsilon)}(s)\right. & \left.-y_{d}(s)\right)^{2} d s \\
\leq K . & \left(\int_{0}^{t} \int_{0}^{s} \mathrm{E}\left|y^{(\varepsilon)}(z)-y(z)\right|^{2} d z+o_{\varepsilon}(t)\right. \\
& +\int_{0}^{T} \sqrt{\mathrm{P}\left(s \notin \bigcup_{n}\left[\sigma_{n}(c), \bar{\sigma}_{n}(b)\right]\right)+\mathrm{P}\left(\sup _{t \in[0, T]}\left|\varphi^{(\varepsilon)}(s)-\varphi(s)\right| \geq b / 3\right)} d s \\
& +\mathrm{E} \sup _{z \in[0, T]}\left(\left|y_{d}^{(\varepsilon)}(z)\right|^{2}+\left|y_{d}(z)\right|^{2}\right) \mathbb{1}_{A_{\varepsilon, r, b, c, \delta, \eta, \varkappa}} \\
& \left.+\operatorname{Esup}_{n}\left|y_{d}^{(\varepsilon)}\left(\sigma_{n}(c)\right)\right|^{2} \mathbb{1}_{\bar{A}_{\varepsilon, r, b, c, \delta, \eta, \varkappa}}+\mathrm{Esup}_{n}\left|y_{d}\left(\sigma_{n}(c)\right)\right|^{2} \mathbb{1}_{\bar{A}_{\varepsilon, r, b, c, \delta, \eta, \varkappa}}\right)
\end{aligned}
$$

where $\sup _{t} o_{\varepsilon}(t) \rightarrow 0$ as $\varepsilon \rightarrow 0+$.

Lemma 3.2. For an arbitrary $\delta>0$, there exists $\eta>0$ such that

$$
\begin{array}{r}
\sup _{\varepsilon \in(0,1)} \mathrm{P}\left(\operatorname { s u p } _ { | s _ { 1 } - s _ { 2 } | < \eta } \left\{\left|\int_{s_{1}}^{s_{2}} a_{0, d}^{\prime}\left(\varphi^{(\varepsilon)}(z)\right) y_{\varepsilon}(z) d z\right|+\left|\int_{s_{1}}^{s_{2}} a_{0, d}^{\prime}(\varphi(z)) y(z) d z\right|\right.\right. \\
+\sum_{k=1}^{m}\left(\left|\int_{s_{1}}^{s_{2}} a_{k, d}^{\prime}\left(\varphi^{(\varepsilon)}(z)\right) y_{\varepsilon}(z) d w_{k}(z)\right|\right. \\
\left.\left.\left.+\left|\int_{s_{1}}^{s_{2}} a_{k, d}^{\prime}(\varphi(z)) y(z) d w_{k}(z)\right|\right)\right\}>\delta\right)<\delta .
\end{array}
$$

The proof of Lemma 3.2 is obtained from the Burkholder inequality, bound (9), and Lemma 3.1.

Lemma 3.3. Assume that the function $z(t)$ is such that

$$
z(t)=z(0)+\int_{0}^{t} f(s) z(s) d s+\varphi(t), \quad t \in[0, T]
$$

where $|z(0)|<c, f(s) \leq 0$ for $s \in[0, T], \sup _{s \in[0, T]}|\varphi(s)|<\delta$, and $\varphi$ is a stepwise continuous function. Then

$$
z(t) \leq c \exp \left\{\int_{0}^{t} f(s) d s\right\}+2 \delta, \quad t \in[0, T] .
$$

The function $z(t)$ can be written explicitly as

$$
\begin{aligned}
z(t)= & \varphi(t)+(z(0)-\varphi(0)) \exp \left\{\int_{0}^{t} f(s) d s\right\} \\
& +\exp \left\{\int_{0}^{t} f(s) d s\right\} \int_{0}^{t} \exp \left\{-\int_{0}^{s} f(z) d z\right\} f(s) \varphi(s) d s \\
\leq & \delta+(c+\delta) \exp \left\{\int_{0}^{t} f(s) d s\right\}-\delta \exp \left\{\int_{0}^{t} f(s) d s\right\} \int_{0}^{t} \exp \left\{-\int_{0}^{s} f(z) d z\right\} f(s) d s \\
= & c \exp \left\{\int_{0}^{t} f(s) d s\right\}+2 \delta .
\end{aligned}
$$

This is what was to be proved. 
Lemma 3.4. Let $\psi \in[0, \infty) \rightarrow[0, \infty)$ be a nonincreasing differentiable function such that $\psi(x)=1$ for $x \in[0,1]$ and $\psi(x)=0$ for $x \in[2, \infty)$. Put $f_{\varepsilon}(x)=\psi(x / \varepsilon) / x^{2}, x>0$. Assume that $x_{t}$ is a nonnegative function such that $\int_{0}^{\Delta} f_{\varepsilon}\left(x_{t}\right) d t \geq \varkappa$.

Then

$$
\int_{0}^{\Delta}\left(\frac{d f_{\varepsilon}}{d x}\right)\left(x_{t}\right) d t \leq-\frac{\varkappa}{\varepsilon} .
$$

The assertion of Lemma 3.4 follows from the estimate

$$
f_{\varepsilon}^{\prime}(x)=\frac{\psi^{\prime}(x / \varepsilon) \varepsilon^{-1} x^{2}-2 \psi(x / \varepsilon) x}{x^{4}} \leq \frac{-2 \psi(x / \varepsilon)}{x^{3}} \leq \frac{-2 f_{\varepsilon}(x)}{x} \leq \frac{-f_{\varepsilon}(x)}{\varepsilon} .
$$

Let $\alpha>0$ and $\delta>0$ be arbitrary. We choose the numbers $r>0, b>0, c>b, \eta>0$, $\varkappa>0$, and $\varepsilon_{0} \in(0, b / 2)$ such that

$$
\int_{0}^{T} \sqrt{\mathrm{P}\left(s \notin \bigcup_{n}\left[\sigma_{n}(c), \bar{\sigma}_{n}(b)\right]\right)+\mathrm{P}\left(\sup _{t \in[0, T]}\left|\varphi^{\varepsilon}(s)-\varphi(s)\right| \geq b / 3\right)} d s<\alpha
$$

and

$$
\mathrm{P}\left(A_{\varepsilon, r, b, c, \delta, \eta, \varkappa)<\alpha}\right.
$$

for all $\varepsilon \in\left[0, \varepsilon_{0}\right]$. This set of numbers exists, since

$$
\mathrm{P}\left(\int_{0}^{t} \mathbb{1}_{\left\{\varphi_{d}(s)=0\right\}} d s=0\right)=1
$$

(see condition (18)) and in view of Lemmas 3.1 and 3.2 and relations (9), (25), and

$$
\mathrm{P}\left(\xi\left(\sigma_{n+1}(c)\right)-\xi\left(\bar{\sigma}_{n}(b)\right)>0 \text { for all } n\right)=1 .
$$

Then

$$
\begin{aligned}
\int_{0}^{t} \mathrm{E}\left(y_{d}^{(\varepsilon)}(s)-y_{d}(s)\right)^{2} d s & \\
\leq K \cdot\left(\int_{0}^{t} \int_{0}^{s} \mathrm{E}\left|y^{(\varepsilon)}(z)-y(z)\right|^{2} d z+o_{\varepsilon}(t)+\alpha\right. & \\
+\left(\mathrm { E } \operatorname { s u p } _ { z \in [ 0 , T ] } \left(\left(y_{d}^{(\varepsilon)}(z)\right)^{2}\right.\right. & \left.\left.+\left(y_{d}(z)\right)^{2}\right)^{2}\right)^{1 / 2} \alpha^{1 / 2} \\
& \left.+\left((\delta+r) e^{-\varkappa / \varepsilon}+2 \delta\right)^{2}+\delta^{2}\right)
\end{aligned}
$$

for all $\varepsilon \in\left(0, \varepsilon_{0}\right)$ by $(26),(27)$, and (28) and Lemmas 3.3 and 3.4. Choosing $\alpha, \delta$, and $\varepsilon_{0}$ sufficiently small, we obtain from (29) and (23) that there exists a number $K>0$ such that, given an arbitrary $\gamma>0$, one can find $\varepsilon_{0}>0$ for which

$$
\begin{gathered}
\mathrm{E}\left|\bar{y}^{(\varepsilon)}(t)-\bar{y}(t)\right|^{2} \leq \gamma+K\left(\int_{0}^{t} \mathrm{E}\left|\bar{y}^{(\varepsilon)}(s)-\bar{y}(s)\right|^{2} d s+\int_{0}^{t} \int_{0}^{s} \mathrm{E}\left|y^{(\varepsilon)}(z)-y(z)\right|^{2} d z d s\right), \\
\int_{0}^{t} \mathrm{E}\left(y_{d}^{(\varepsilon)}(s)-y_{d}(s)\right)^{2} d s \leq \gamma+K \int_{0}^{t} \int_{0}^{s} \mathrm{E}\left|y^{(\varepsilon)}(z)-y(z)\right|^{2} d z d s
\end{gathered}
$$


for all $\varepsilon \in\left(0, \varepsilon_{0}\right)$ and for all $t \in[0, T]$. Then the Gronwall lemma implies that

$$
\begin{array}{rlrl}
\sup _{t \in[0, T]} \mathrm{E}\left|\bar{y}^{(\varepsilon)}(t)-\bar{y}(t)\right|^{2} \rightarrow 0, & \varepsilon & \rightarrow 0+, \\
\int_{0}^{T} \mathrm{E}\left(y_{d}^{(\varepsilon)}(t)-y_{d}(t)\right)^{2} d t \rightarrow 0, & \varepsilon \rightarrow 0+, \\
\int_{0}^{T} \mathrm{E}\left|y^{(\varepsilon)}(t)-y(t)\right|^{2} d t \rightarrow 0, & \varepsilon \rightarrow 0+.
\end{array}
$$

Reasoning in the same way as in the proof of (29) we see that for all $t \in[0, T]$,

$$
\mathrm{E}\left(y_{d}^{(\varepsilon)}(t)-y_{d}(t)\right)^{2} \rightarrow 0, \quad \varepsilon \rightarrow 0+,
$$

by (30) and (24). Theorem 3.1 is proved.

Applying the argument of Section IV in [6], one can check that the processes $\varphi_{t}^{(\varepsilon)}(x)$ are differentiable in the Sobolev sense with respect to the parameter $x$. Moreover

$$
\frac{\partial \varphi_{t}^{(\varepsilon)}(x)}{\partial x}=y_{t}^{(\varepsilon)}(x) \quad \text { almost surely, }
$$

where the processes $y_{t}^{(\varepsilon)}(x)$ satisfy equation (17). The processes $y_{t}^{(\varepsilon)}(x)$ have a version that is measurable with respect to $(\omega, t, x)$. According to (16), (19), and (21) we obtain that for almost all $\omega$ the function $y_{t}(\cdot, \omega)$ is the derivative in the Sobolev sense (with respect to $x$ ) of the process $\varphi_{t}(x)$ if $y$ has a version that is measurable with respect to $(\omega, t, x)$.

The corresponding result and the existence of a set $\Omega_{0}$ of full measure such that $y_{t}(x)=\partial \varphi_{t}(x) / \partial x$ for all $t \in[0, T]$ and $\omega \in \Omega_{0}$ are proved in the following theorem.

Theorem 3.2. Assume that the hypotheses of Theorem 3.1 hold. Then

$1)$ there exists a version of the process $y_{t}(x)$ that is measurable with respect to $(t, x, \omega)$;

2) there exists a set $\Omega_{0}, \mathrm{P}\left(\Omega_{0}\right)=1$, such that $y_{t}=\partial \varphi_{t} / \partial x$ is the Sobolev derivative for all $\omega \in \Omega_{0}$ and $t \in[0, T]$.

Proof. We need the following auxiliary result on measurability of the limit stochastic process.

Theorem $3.3([4)$. Let $(\Omega, \mathcal{F}, \mathrm{P})$ be a probability space, $(A, \mathcal{A})$ a measurable space, $Y$ a separable metric space, and let $X_{n}=X_{n}(a), n \geq 1, a \in A$, be a sequence of $\mathcal{F} \otimes \mathcal{A}$ measurable random elements with values in $Y$. Assume that the limit in probability exists for the sequence $\left\{X_{n}(a), n \geq 1\right\}$ and for $a \in A$.

Then there is an $\mathcal{F} \otimes \mathcal{A}$-measurable random element $X(a), a \in \mathcal{A}$, with values in $Y$ such that

for all $a \in \mathcal{A}$.

$$
X_{n}(a) \stackrel{\mathrm{P}}{\longrightarrow} X(a), \quad n \rightarrow \infty
$$

Let $\widetilde{y}_{t}^{c}(x), c>0$, be a sequence approximating the process $y_{t}(x)$ (see (11) and (12)). Theorem 2.1 implies the convergence

$$
\mathrm{E} \sup _{t \in[0, T]}\left|\widetilde{y}_{t}^{c}(x)-y_{t}(x)\right|^{p} \rightarrow 0, \quad c \rightarrow 0+,
$$

for all $x \in \mathbb{R}_{+}^{d}$ and all $p>1$. For a space $Y$ in Theorem 3.3, take the space $D_{[0, T]}$ of right continuous functions on $[0, T]$ that have left limits. It follows from Theorem 3.3 that to complete the proof of the first part of Theorem 3.2, it suffices to verify measurability of some version of the processes $\widetilde{y}_{t}^{c}(x)$ with respect to $(x, \omega)$. 
Similarly to (4) and (5) we introduce the stopping times

$$
\begin{aligned}
\tau_{0}(c, x) & =\inf \left\{t \geq 0: \varphi_{t}^{d}(x)=0\right\}, \quad x \in \mathbb{R}_{+}^{d}, \\
\sigma_{j}(c, x) & =\inf \left\{t \geq \tau_{j}(c, x): \varphi_{t}^{d}(x)=c\right\}, \\
\tau_{j+1}(c, x) & =\inf \left\{t \geq \sigma_{j}(c, x): \varphi_{t}^{d}(x)=0\right\} .
\end{aligned}
$$

The random elements $\sigma_{j}(c, x)$ and $\tau_{j}(c, x)$ are measurable with respect to $(x, \omega)$ for all $c>0$, since $\varphi_{t}(x)$ is continuous with respect to $(t, x)$.

Measurability of $\widetilde{y}_{t}^{c}(x)$ can be checked by using Theorem 3.3 again, since the process $\widetilde{y}_{t}^{c}(x)$ is the limit of successive approximations and the stochastic integrals in the successive approximations are limits of integral sums. The first part of Theorem 3.2 is proved.

Relations (16), (19), and (31) and Lemma 3.1 imply that

$$
\frac{\partial \varphi_{t}}{\partial x}=y_{t} \quad \text { in } L_{p, \text { loc }}\left(\mathbb{R}_{+}^{d}\right)
$$

for all $t \in[0, T]$ and almost all $\omega$. Since the process $y_{t}(x)$ has right continuous trajectories, relations (15), (22), and (32) imply that for all $\omega$ belonging to a set of full measure and for all $r>0$ and $t \in[0, T]$,

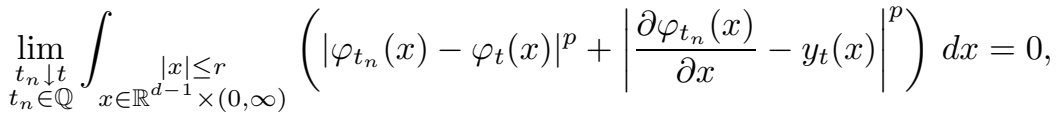

that is, $y_{t}=\partial \varphi_{t} / \partial x, t \in[0, T]$, almost surely.

Theorem 3.2 is proved.

\section{BIBLIOGRAPHY}

1. A. Yu. Pilipenko, Flows generated by stochastic equations with reflection, Random Oper. Stochastic Equations 12 (2004), no. 4, 389-396. MR2108191(2006a:60109)

2. A. Yu. Pilipenko, Properties of flows generated by stochastic equations with reflection, Ukrain. Mat. Zh. 57 (2005), no. 8, 1069-1078; English transl. in Ukrainian Math. J. 57 (2005), no. 8, 1262-1274. MR2218469 (2007g:60066)

3. H. Tanaka, Stochastic differential equations with reflecting boundary condition in convex regions, Hiroshima Math. J. 9 (1979), no. 1, 163-177. MR529332(80k:60075)

4. C. Striker and M. Yor, Calcul stochastique dépendant d'un paramètre, Z. Wahrsch. Verw. Gebiete 45 (1978), no. 2, 109-133. MR.510530 (80f:60047)

5. P. Billingsley, Convergence of Probability Measures, John Wiley \& Sons, Inc., New YorkLondon-Sydney-Toronto, 1968. MR0233396 (38:1718)

6. N. Bouleau and F. Hirsch, Dirichlet Forms and Analysis on Wiener Space, de Gruyter Studies in Mathematics, vol. 14, Walter de Gruyter \& Co., Berlin, 1991. MR.1133391 (93e:60107)

7. A. Jakubowski, A non-Skorokhod topology on the Skorokhod space, Electron. J. Probab. 2 (1997), no. 4. MR1475862 (98k:60046)

Institute of Mathematics, National Academy of Sciences of Ukraine, Tereshchenkivs'ka Street, 3, 01601, Kyiv, Ukraine

E-mail address: apilip@imath.kiev.ua

Received 19/NOV/2004

Translated by O. I. KLESOV 